\title{
Case mix at the European Institute of Oncology: first report of the Tumour Registry, 2000-2002
}

\author{
E Botteri, S lodice, P Maisonneuve, M Alfieri, N Burzoni, L Manghi, M Martinetti, B Montanari, E Albertazzi, B Bazolli and N \\ Rotmensz
}

Division of Epidemiology and Biostatistics, European Institute of Oncology, via Ripamonti 435, Milan, Italy

\begin{abstract}
Introduction: An institutional and centralized hospital-based tumour registry (TR) is the ideal supporting tool for the organization and management of clinical data in a comprehensive cancer centre. The purpose of this paper is to describe the development of the TR at the European Institute of Oncology (IEO), Milan, Italy, from its origin to its current applications.

Material and methods: After a series of meetings with members of administrative, clinical, research and informatics departments, the TR was activated in March 2006 with the aim of collecting data on all the individuals referred to the institute, with or at risk of developing a tumour. It was implemented on an Oracle ${ }^{\mathrm{TM}}$-based interface. A minimum dataset of variables was defined and data collection was divided into four forms, which together gather all the relevant data on patients, tumours, treatments and subsequent events.
\end{abstract}

Results: After a six-month pilot period, which involved the training of the tumour registrars, adjustments to the structure of the registry, development of a data quality control procedure and finalization of the operative protocol, since September 2006 the data collection has been fully operative. Five registrars have been chronologically entering data of all individuals who visited the IEO for the first time since 1 January 2000. As of March 2009, data on 69,637 individuals and 43,567 tumours has been reviewed, recoded and registered in the TR. Twenty-two per cent of the tumours $(n=9578)$ were first invasive primaries, diagnosed and treated in the IEO; the most common sites were breast $(n=4972)$, lung $(n=627)$, intestines $(n=479)$ and prostate $(n=376)$.

Conclusion: The IEO TR has been proven functional and reliable in monitoring the activity of the hospital, allowing extraction of data from any subpopulation with characteristics of interest. The structured and centralized TR represents an important tool for our researchoriented institution.

Published: $21 / 10 / 2009$

Received: $26 / 06 / 2009$

ecancer 2009, 3:149 DOI: 10.3332/ecancer.2009.149

Copyright: (C) the authors; licensee ecancermedicalscience. This is an Open Access article distributed under the terms of the Creative Commons Attribution License (http://creativecommons.org/licenses/by/2.0), which permits unrestricted use, distribution, and reproduction in any medium, provided the original work is properly cited.

Competing Interests: The authors have declared that no competing interests exist.

Correspondence to E Botteri. Email: edoardo.botteri@ieo.it 


\section{Introduction}

The European Institute of Oncology (IEO) aims at excellence in the prevention, diagnosis and treatment of cancer through highlevel clinical and scientific research. Since its opening in 1994, the number of individuals presenting for the first time to the IEO has increased year by year, reaching 38,500 in 2008 . Thus, the need of a well-structured and centralized institutional database has become essential. Furthermore, to optimize the use of the great quantity of informa tion, some obstacles typical of the organization and management of hospital data had to be overcome, such as the presence of many non-standardized databases, duplication of information and absence of centralized follow-up. For these reasons, the IEO tumour registry (TR) was planned, designed and finally activated in 2006.

The purposes of a hospital-based TR are to support the administration of the hospital, back up the clinical and scientific research and, above all, serve the needs of the patient [1]. In fact a TR can provide immediate reports on the activity of the hospital, document the cancer burden borne by the hospital for specific periods of time, provide background information useful for the design of clinical studies, extract data of any subpopulation with characteristics of interest and organize a centralized follow-up of patients, avoiding duplication of information and useless contact between the hospital and the patients.

The aims of the present paper are to illustrate the development of the IEO TR, from its origin to its current applications, and to describe the case-mix of our institute. We will report data from its first three years of activity, including data on all individuals presenting to the institute between 2000 and 2002, already having or being at risk of developing a tumour.

\section{Material and methods}

\section{Brief history}

The TR project was developed in many successive phases, starting with a series of meetings and discussions on 'who' an 'what' to include. A group of experts, consisting of physicians, information technology (IT) specialists, data managers, administrative personnel and biostatisticians had regularly met for almost two years to discuss the format and the data to be collected. An important part of the planning was also dedicated to visiting and contacting other hospital-based cancer registries, in order to learn from already established realities. Thereafter, the database was designed and tested. It was decided to implement the TR using an Oracle ${ }^{\mathrm{TM}}$-based interface. The implementation, completed in February 2006, was managed in the Division of Epidemiology and Biostatistics, with the help of the IT division. The next phase involved the selection and training of dedicated data registrars. The project finally got off the ground in March 2006, and at present five dedicated persons are working full time on entering the data. Data quality control and data management are carried out by two data managers and two biostatisticians.

\section{Eligibility criteria}

We agreed to collect information on all individuals presenting at the IEO since its opening, either with a tumour or at risk of developing one. We also decided to collect information on patients with previous invasive tumours, either treated at this institute or elsewhere. In order to gain entry in the TR, patients must have: (1) the IEO unique identification number, assigned to the individual at the first visit; and (2) at least one medical report (i.e., pathological report or diagnostic examination) accessible from the institute's intranet.

\section{Information collected}

A minimum dataset of variables was defined, to establish the best compromise between synthesis and informativeness. Data collection is divided into four forms: on the first form personal data (i.e. sex, date of birth) and information on survival (i.e. date of last contact, date of last visit, vital status, cause of death) are recorded. The type of record is registered in this form. The following types of record are assigned to each individual.

1. Visit: a healthy individual comes to the IEO for either a visit or a genetic counselling.

2. Anamnesis: the patient, at the moment free of disease, reports on a tumour diagnosed in the past and already treated and cured elsewhere.

3. Diagnosis: diagnosis of tumour is made at the IEO and the patient decides to be treated and followed up elsewhere.

4. Second opinion: the patient or the patient's parents come to the IEO and ask for an opinion on a diagnosis and/or a treatment proposed elsewhere. 


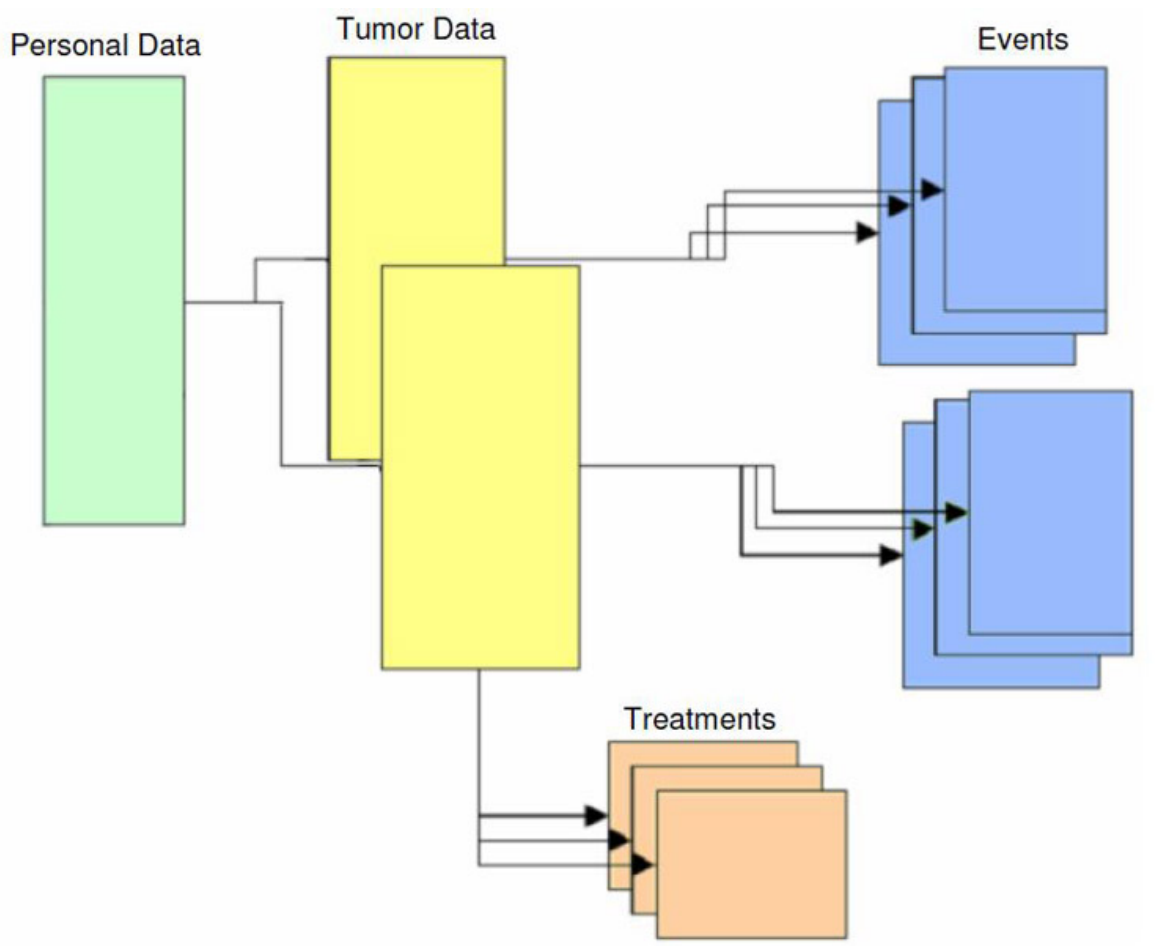

Figure 1: The structure of the IEO tumour registry

5. Long: the patient receives at least one treatment at the IEO.

Detailed information on the patient's tumour(s) (i.e. date of diagnosis, morphology, topography, TNM staging, ordinal number of the tumour for multiple tumours) is recorded on the second form, together with some epidemiological information (i.e. familiarity, height and weight at diagnosis and smoking habits). The third form is dedicated to the treatment strategy, where every therapy is classified as administered or proposed. The fourth catalogues, chronologically, all following events, tracing the whole history of the disease.

It is important to note that the amount of data collected will vary according to the type of record: the completion of all four forms only occurs for patients coded as 'Long'. For patients coded as 'Anamnesis', 'Diagnosis' or 'Second opinion' only the personal data form together with date of diagnosis, place of diagnosis, the IEO histopathological identification code (if any), topography and morphology are registered. For patients coded as 'Visit' only the personal data form is completed.

Invasive and in situ tumours are always collected, whereas benign tumours or negative histologies are collected only if the patient undergoes a major surgical procedure in the IEO.
Examples of benign tumours/negative histologies we collect are: adenoma of the thyroid after thyroidectomy, fibroadenoma after quadrantectomy and negative histology after hysterectomy.

The structure of the TR is represented in Figure 1. The patient is the basis of the structure. Zero, one or more tumours can be linked to the patient. Zero, one or more treatments and events can be linked to each tumour.

Systematized Nomenclature of Medicine-Clinical Terms (SNOMED CT) [2] is used to classify tumours according to topography and morphology except for haematopoietic tumours, for which we use the World Health Organization (WHO) ICD-10 classification [3]. Clinical and pathological staging is based on the TNM system [4], whether the fifth or the sixth edition according to the date of diagnosis. Cause of death is based on the WHO ICD-10 classification [3].

\section{Sources of data, data entry and quality control}

The IEO TR data entry is completely paperless, as all the information derives from computerized sources: the IEO patients' administrative database (personal information is automatically visible on the personal data form), medical reports 
accessible on the IEO intranet, online databases (surgery, laboratory medicine, pathology, etc.) and patients' clinical dossier, which are all digitalized and accessible online.

Five data registrars work full time on data entry. Each one has specialized in a particular tumour or set of tumours in order to achieve specific knowledge and abilities. Data quality control and data management are carried out by two data managers and two biostatisticians. A data manager randomly reviews data entered by a registrar, and the two discuss potential mistakes. A field was created in which notes regarding which cases are revised by a data manager and which are not inserted. Furthermore, in order to clarify any doubt on specific cases, a medical doctor was chosen from each division as a referent to address questions to.

\section{Results}

A six-month pilot period (March to August 2006) was necessary to train the registrars, adjust the structure of the registry, develop a data quality control procedure and edit the operative protocol. Since September 2006, the data entry has been running at top speed. We started by entering individuals who presented for the first time to the IEO in the year 2000 in a sequential fashion. By March 2009, 69,637 individuals had been entered in the TR, corresponding to individuals who came for the first time to the IEO between 2000 and 2002, not necessarily presenting with a tumour. In Table 1, some characteristics of these individuals are reported. Approximately, one out of four was classified as 'Long', as he/she received at least one treatment in the IEO. Only $1.6 \%$ of individuals were 19 years old or less, as our institute does not treat paediatric tumours.

Overall, 39,480 individuals out of 69,637 had one or more tumours. Information on a total of 43,567 tumours was collected, with an average of 1.1 tumours per person. Frequencies by site, malignancy, place of diagnosis and place of surgery are reported in Table 2.

In Table 3, we reported only the first invasive primary tumours, diagnosed and treated in the IEO $(n=9578)$, which represent the typical subgroup of tumours used for epidemiological retrospective studies. Breast was the most frequent tumour site by far $(n=4972)$, followed by lung $(n=627)$, intestines $(n=479)$ and prostate $(n=376)$. In order to show an example of the reports that can be easily obtained from the TR, we included some additional information for the most frequent tumour sites: breast (women only), colorectal, lung, non-Hodgkin lymphoma, ovary, prostate, sarcoma, skin melanoma and tongue (Appendices A-I].

Finally, we calculated that, on average, it took about ten minutes to enter a case, that is six cases per hour. The time varied depending especially on the type of record: 22 minutes for a long case, two minutes for a visit and nine minutes for other types of record. By the end of 2009, data of all individuals who referred to the IEO for the first time in the years 2000-4 will be collected, for a total of about 125,000 individuals and 80,000 tumours.

\section{Discussion}

This paper presents the first three years of activity of the IEO TR, specifically data on all individuals presenting for the first time to the institute in the years 2000-2, already having or being at risk of developing a tumour. This first analysis of the TR data demonstrated that the registry can support the administration in monitoring the hospital activity, be a supporting tool for the clinical practice as well as for epidemiological/basic research, play a key role in the production of publications on any subpopulation with characteristics of interest and, above all, improve patient management and knowledge of the disease.

Regarding information on events and survival, although patients in the TR had a theoretically adequate follow-up, with a median of six years, we preferred not to report any result, as we have not performed a detailed analysis of the quality of the follow-up yet. By now, vital status (dead/alive) and events are based on passive follow-up, with an accuracy depending on the site or other characteristics of the tumours. For example, since our institute produces many publications in breast cancer field, follow-up information on patients suffering from that tumour is updated more frequently compared to patients with other tumours. Survival analyses on different tumours might have been incomparable. By 2010, after a detailed analysis of the follow-up information collected in our institute, an active centralized follow-up will be organized and started.

Although it was decided that researchers or clinicians would not have direct access to the TR, we have established a system to collect and answer requests. Data extrapolation and analysis are managed in the Division of Epidemiology and Biostatistics.

A recent paper entitled 'Analysis of local and regional recurrences in breast cancer after conservative surgery' represents the first study conducted on the data of the TR [ $\underline{5}$. It consisted of a multistage analysis of local, regional and distant 


\begin{tabular}{|c|c|}
\hline Classification & $\begin{array}{c}\text { No. of Patients } \\
(\%)\end{array}$ \\
\hline \multicolumn{2}{|l|}{ Gender } \\
\hline Male & $20,941(30.1)$ \\
\hline Female & 48,696 (69.9) \\
\hline \multicolumn{2}{|l|}{ Age } \\
\hline$<20$ years & $985(1.6)$ \\
\hline 20-34 years & 6909 (10.8) \\
\hline $35-49$ years & $19,052(28.1)$ \\
\hline $50-64$ years & 24,406 (32.8) \\
\hline $65-79$ years & $16,310(24.3)$ \\
\hline $80+$ years & $1975(2.4)$ \\
\hline \multicolumn{2}{|l|}{ Place of residence } \\
\hline Northern Italy & $46,940(67.4)$ \\
\hline Lombardy & 39,207 (56.3) \\
\hline Milan & $14,679(21.1)$ \\
\hline Central Italy & $12,138(17.4)$ \\
\hline Southern Italy & $10,211(14.7)$ \\
\hline Foreign countries & $348(0.5)$ \\
\hline \multicolumn{2}{|l|}{ Type of record } \\
\hline Long & $17,823(25.6)$ \\
\hline Second Opinion & $19,794(28.4)$ \\
\hline Anamnesis & $1095(1.6)$ \\
\hline Diagnosis & 768 (1.1) \\
\hline Visit & $30,157(43.3)$ \\
\hline Total & $69,637(100.0)$ \\
\hline
\end{tabular}

Individuals who presented at the IEO for the first time in years 2000-2002.

recurrences, performed on data of 2784 women treated for early breast cancer by quadrantectomy and whole breast irradiation at the IEO. The authors concluded that 'local and regional recurrences after breast-conserving surgery are rare events. They are markers of tumour aggressiveness and indicators of an increased likelihood of distant metastases'.
As shown in this paper, we can easily monitor the activity of the IEO and extrapolate data from any subpopulation with characteristics of interest. We strongly believe that a wellstructured and centralized TR is a necessary tool for our, and in fact any, institution where research is a fundamental part of its activity. 
Table 2: Tumours by site

\begin{tabular}{|c|c|c|c|c|}
\hline \multirow{2}{*}{ Tumour She } & \multirow{2}{*}{$\begin{array}{l}\text { Collected } \\
\text { Overall" }\end{array}$} & \multicolumn{3}{|c|}{ Invasive Tumours } \\
\hline & & Total & Diagnosis & Surgery \\
\hline Head and neck & 1294 & 1121 & $340(30.3)$ & $249(22.2)$ \\
\hline up & 54 & 47 & $13(27.7)$ & $12(25.5)$ \\
\hline Tongus & 235 & 224 & $104(46.4)$ & $93(41.5)$ \\
\hline Majpr watkary gands & 253 & 135 & $49(36.3)$ & $33(24.4)$ \\
\hline oum & 15 & 13 & $7(53.8)$ & $7(53.8)$ \\
\hline Fibor ef mouth & 37 & 34 & $5(14.7)$ & $5(14.7)$ \\
\hline Other and unspecited parts of mouth & 210 & 192 & $66(34,4)$ & $53(27.6)$ \\
\hline Oropherynx & 155 & 149 & $28(18.8)$ & $18(12.1)$ \\
\hline Nesophaynx & 142 & 141 & $28(19.9)$ & $5(3.5)$ \\
\hline Hipophaynix & 87 & 87 & $13(14.9)$ & $10(11.5)$ \\
\hline Othere and a defoned sans & 106 & 99 & $27(27.3)$ & $13(13.1)$ \\
\hline Digentive organs and pertioneumr & 6435 & 6334 & $1215(19.2) 6$ & $693(10.9)$ \\
\hline Oesophagus & 185 & 181 & $46(25.4)$ & $19(10.5)$ \\
\hline stomech & 1119 & 1109 & $228(20.6) \quad 1$ & $161(14.5)$ \\
\hline Smat ichastree, eccusng duodenum & 109 & 105 & $25(23.8)$ & $2(1.9)$ \\
\hline colon & 2324 & 2289 & $418(18.3) \quad 2$ & $277(121)$ \\
\hline Rectum, rectosigmoid junction and anus & 991 & 977 & 240 (24.6) & $179(18.3)$ \\
\hline Liver and intra-hepatcic bie ducts & 484 & 476 & $75(15.8)$ & $16(3.4)$ \\
\hline 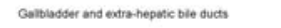 & 336 & 324 & $35(10.8)$ & $6(1.9)$ \\
\hline Pancreas & 771 & 766 & $120(15.7)$ & $28(3.7)$ \\
\hline Retropentonowum and pertioneuum & 56 & 52 & $19(36.5)$ & $4(7.7)$ \\
\hline Oreser and aldefined atest & 60 & 55 & $9(16.4)$ & $1(1.8)$ \\
\hline Respiratory and intra-thoracic organs & 5068 & 4853 & $1297(26.7)$ & $811(16.7)$ \\
\hline $\begin{array}{l}\text { Nasol canties, misto ear and accossory } \\
\text { nnuses }\end{array}$ & 32 & 31 & $2(6.5)$ & $0(0.0)$ \\
\hline Largnx & 622 & 547 & $188(34.4)$ & $167(30.5)$ \\
\hline Tractsen, brenchus and wing & 4097 & 3983 & $1014(25.5)$ & $610(15.3)$ \\
\hline Plecra & 191 & 181 & $53(29.3)$ & $10(5.5)$ \\
\hline Thrmus meart and mediastrum & 123 & 108 & $40(37.0)$ & $24(22.2)$ \\
\hline Other and a-detined shes & 3 & 3 & $0(0.0)$ & $0(0.0)$ \\
\hline Bone, connective tisuve, skin and breast & 18871 & 15305 & $7907(51.7) 5$ & $5396(35.3)$ \\
\hline Bone and anticulat catiage & 12 & 8 & $1(12.5)$ & $0(0.0)$ \\
\hline Connective and other with bsuve & 954 & 897 & $383(42.7)$ & $156(17.4)$ \\
\hline $\operatorname{sen}^{4}$ & 2280 & 2160 & $1003(46.4)$ & $626(29.0)$ \\
\hline Breast & 15,625 & 12240 & $6520(53.3) 4$ & $4614(37.7)$ \\
\hline Genitourinary organs & 8032 & 6694 & $1799(26.9)$ & $1192(17.8)$ \\
\hline Convis vater & 1344 & 559 & $218(39.0)$ & 156 (27.9) \\
\hline Uterine cospus & 618 & 525 & $164(31.2)$ & $116(22.1)$ \\
\hline Orary and other uterine adnexs & 1222 & 1099 & $398(36.2)$ & $202(18.4)$ \\
\hline Onere and unspected tormalo genatal cepans & 250 & 176 & $73(41.5)$ & $46(26.1)$ \\
\hline Prostate & 2378 & 2334 & $504(21.6)$ & $333(14.3)$ \\
\hline Testin & 281 & 261 & $75(28.7)$ & $50(19.2)$ \\
\hline Penis and ofther male gental organs & 44 & 36 & $11(30.6)$ & $11(30.6)$ \\
\hline Blossor & 953 & 807 & $172(21.3)$ & $137(17.0)$ \\
\hline kotery & 833 & 801 & $171(21.3)$ & $131(16.4)$ \\
\hline Other and aldetined sten & 109 & 96 & $13(13.5)$ & $10(10.4)$ \\
\hline Other and unspecified sites & 1631 & 1355 & $220(16.2)$ & $169(12.5)$ \\
\hline Eyo & 6 & 5 & $1(20.0)$ & $0(0.0)$ \\
\hline Brain & 716 & 703 & $21(3.0)$ & $0(0.0)$ \\
\hline Coser and unspected parts of nevvous system & 16 & 15 & $0(0.0)$ & $0(0.0)$ \\
\hline Thyoid giand & 825 & 583 & $190(32.6)$ & 164 (28.1) \\
\hline Coser endocine glands and related structure & 68 & 49 & $8(16.3)$ & $5(10.2)$ \\
\hline Lymphabic and hasematopoietetic tissue & 1405 & 1395 & $554(39.7)$ & $28(2.0)$ \\
\hline Hodgken inmehoma & 238 & 237 & $96(40.5)$ & $3(1.3)$ \\
\hline Non-Hosgkin ymphoma & 780 & 777 & $373(48.0)$ & ) $25(3.2)$ \\
\hline Mubple mpoloma & 171 & 171 & $46(26.9)$ & $0(0.0)$ \\
\hline Leukbermia & 203 & 203 & $36(17.7)$ & $0(0.0)$ \\
\hline Cosere and unspecties & 13 & 7 & $3(42.9)$ & $0(0.0)$ \\
\hline Non-skin melanoma & 51 & 51 & $16(31.4)$ & $12(23.5)$ \\
\hline Malignant neoplasm withoua speciscation of atto & 780 & 767 & $206(26.9)$ & $\begin{array}{c}23(3.0) \\
8573\end{array}$ \\
\hline Total & & & (35.8 & 8) (22.6) \\
\hline
\end{tabular}

Table 3: First invasive primaries diagnosed and treated in the IEO ${ }^{\mathrm{a}}$ by site, gender and age 
ecancer 2009, 3:149

\begin{tabular}{|c|c|c|c|c|c|c|c|c|}
\hline & \multirow{2}{*}{ All } & \multicolumn{2}{|c|}{ Gender } & \multicolumn{5}{|c|}{ Age Distribution (years) } \\
\hline & & Female & Male & $<35$ & $35-49$ & $50-64$ & 65.79 & 80 \\
\hline Head and neck & 248 & $82(33.1)$ & $166(66.9)$ & $28(11.3)$ & $35(14.1)$ & $101(40.7)$ & $74(29.8)$ & $10(4.0)$ \\
\hline Lip & 10 & $2(20.0)$ & $8(80.0)$ & $0(0,0)$ & $0(0.0)$ & $1(10.0)$ & $7(70.0)$ & $2(20.0)$ \\
\hline Tongue & 90 & $34(37.8)$ & $56(62.2)$ & $12(13.3)$ & $14(15.6)$ & $34(37.8)$ & $25(27.8)$ & $5(5.6)$ \\
\hline Major salikary glands & 31 & $15(48.4)$ & $16(51.6)$ & $10(32.3)$ & $2(6.5)$ & $15(48.4)$ & $3(9.7)$ & $1(3.2)$ \\
\hline Gum & 5 & $2(40.0)$ & $3(60.0)$ & $1(20.0)$ & $0(0.0)$ & $0(0.0)$ & $4(80.0)$ & $0(0.0)$ \\
\hline Floor of mouth & 4 & $2(50.0)$ & $2(50.0)$ & $0(0.0)$ & $3(75.0)$ & $1(25.0)$ & $0(0.0)$ & $0(0.0)$ \\
\hline Other and unspecified parts of mouth & 48 & $11(22.9)$ & $37(77.1)$ & $0(0.0)$ & $8(16.7)$ & $22(45.8)$ & $16(33.3)$ & $2(4.2)$ \\
\hline Oropharynix & 22 & $6(27.3)$ & $16(72.7)$ & $2(9.1)$ & $2(9.1)$ & $10(45.5)$ & $8(36.4)$ & $0(0.0)$ \\
\hline Nasopharynx & 13 & $3(23.1)$ & $10(76.9)$ & $3(23,1)$ & $3(23.1)$ & $4(30.8)$ & $3(23.1)$ & $0(0.0)$ \\
\hline Oropharynx Hypopharynx & 10 & $2(20.0)$ & $8(80.0)$ & $0(0.0)$ & $1(10.0)$ & $5(50.0)$ & $4(40.0)$ & $0(0.0)$ \\
\hline Other and in deffned sites & 15 & $5(33.3)$ & $10(66.7)$ & $0(0.0)$ & $2(13.3)$ & $9(60.0)$ & $4(26.7)$ & $0(0.0)$ \\
\hline Digestive organs and peritoneum & 791 & $\begin{array}{l}370 \\
(46.8)\end{array}$ & $421(53.2)$ & $19(2.4)$ & $111(14)$ & $331(41.8)$ & $294(37.2)$ & $36(4.6)$ \\
\hline Oesophagus & 20 & $9(45.0)$ & $11(55.0)$ & $0(0.0)$ & $4(20.0)$ & $10(50.0)$ & $5(25.0)$ & $1(5.0)$ \\
\hline Stomach & 166 & $68(41.0)$ & $98(59.0)$ & $5(3.0)$ & $27(16.3)$ & $67(40.4)$ & $57(34.3)$ & $10(6.0)$ \\
\hline Small intestine, including duodenum & 11 & $4(36.4)$ & $7(63.6)$ & $0(0.0)$ & $4(36.4)$ & $5(45.5)$ & $2(18.2)$ & $0(0.0)$ \\
\hline Colon & 282 & $\begin{array}{l}146 \\
(51.8)\end{array}$ & $136(48.2)$ & $7(2.5)$ & $35(12.4)$ & $115(40.8)$ & $109(38.7)$ & $16(5.7)$ \\
\hline $\begin{array}{l}\text { Rectum, rectosigmoid junction and } \\
\text { anus }\end{array}$ & 186 & $74(39.8)$ & $112(60.2)$ & $2(1.1)$ & $21(11.3)$ & $83(44.6)$ & $71(38.2)$ & $9(4.8)$ \\
\hline Liver and intra-hepatic bile ducts & 31 & $10(32.3)$ & $21(67.7)$ & $0(0.0)$ & $1(3.2)$ & $11(35.5)$ & $19(61.3)$ & $0(0.0)$ \\
\hline $\begin{array}{l}\text { Galbladder and extra-hepatc ble } \\
\text { ducts }\end{array}$ & 18 & $7(38.9)$ & $11(61.1)$ & $0(0.0)$ & $7(38.9)$ & $6(33.3)$ & $5(27.8)$ & $0(0.0)$ \\
\hline Pancieas & 70 & $47(67.1)$ & $23(32.9)$ & $3(4.3)$ & $10(14.3)$ & $31(44.3)$ & $26(37.1)$ & $0(0.0)$ \\
\hline Retroperitoneum and peritoneum & 5 & $3(60.0)$ & $2(40.0)$ & $2(40.0)$ & $1(20.0)$ & $2(40.0)$ & $0(0.0)$ & $0(0.0)$ \\
\hline Other and il-defined shes & 2 & $2(100.0)$ & $0(0.0)$ & $0(0.0)$ & $1(50.0)$ & $1(50.0)$ & $0(0.0)$ & $0(0.0)$ \\
\hline Respiratory and intra-thoracic organs & 847 & $188(22.2)$ & $659(77.8)$ & $20(2.4)$ & $73(8.6)$ & $355(41.9)$ & $379(44.7)$ & $20(2.4)$ \\
\hline Larynx & 169 & $12(7.1)$ & $157(92.9)$ & $1(0.6)$ & $11(6.5)$ & $75(44.4)$ & $76(45.0)$ & $6(3.6)$ \\
\hline Trachea, bronchus and lung & 627 & $157(25.0)$ & $470(75.0)$ & $13(2.1)$ & $49(7.8)$ & $259(41.3)$ & $293(46.7)$ & $13(2.1)$ \\
\hline Pleura & 20 & $9(45.0)$ & $11(55.0)$ & $0(0.0)$ & $3(15.0)$ & $11(55.0)$ & $5(25.0)$ & $1(5.0)$ \\
\hline Thymus, heart and mediastinum & 31 & $10(32.3)$ & $21(67.7)$ & $6(19.4)$ & $10(32.3)$ & $10(32.3)$ & $5(16.1)$ & $0(0.0)$ \\
\hline Bone, connective tissue, skin and breast & 5837 & $5370(92)$ & $467(8)$ & $320(5.5)$ & $2002(34.3)$ & $2274(39)$ & $1105(18.9)$ & $136(2.3)$ \\
\hline Bone and articular cartilige & 1 & $1(100.0)$ & $0(0.0)$ & $0(0.0)$ & $0(0.0)$ & $0(0.0)$ & $1(100.0)$ & $0(0.0)$ \\
\hline Connective and other son tissue & 180 & $97(53.9)$ & $83(46.1)$ & $26(14.4)$ & $69(38.3)$ & $45(25)$ & $38(21.1)$ & $2(1.1)$ \\
\hline Skin & 684 & $330(48.2)$ & $354(51,8)$ & $62(9.1)$ & $162(23.7)$ & $223(32.6)$ & $201(29.4)$ & $36(5.3)$ \\
\hline Breast & 4972 & $4942(99.4)$ & $30(0.6)$ & $232(4.7)$ & $1771(35.6)$ & $2006(40.3)$ & $865(17.4)$ & $98(2.0)$ \\
\hline Gentourinary organs & 1261 & $629(49.9)$ & $632(50.1)$ & $99(7.9)$ & $210(16.6)$ & $513(40.7)$ & $408(32.4)$ & $31(2.4)$ \\
\hline Conve uteri & 186 & $186(100.0)$ & $0(0.0)$ & $26(14.0)$ & $81(43.5)$ & $59(31.7)$ & $19(10.2)$ & $1(0.5)$ \\
\hline Uierine corpus & 99 & $99(100.0)$ & $0(0.0)$ & $2(2.0)$ & $10(10.1)$ & $57(57.6)$ & $23(23.2)$ & $7(7.1)$ \\
\hline Ovary and other uterine adnexa & 243 & $243(100.0)$ & $0(0.0)$ & $24(9.9)$ & $68(28.0)$ & $109(44.9)$ & $41(16.9)$ & $1(0.4)$ \\
\hline Other and unspecified female gental organs & 51 & $51(100.0)$ & $0(0.0)$ & $0(0.0)$ & $8(15.7)$ & $13(25.5)$ & $25(49.0)$ & $5(9.8)$ \\
\hline Prostate & 376 & $0(0.0)$ & $376(100.0)$ & $0(0.0)$ & $1(0.3)$ & $172(45.7)$ & $198(52.7)$ & $5(1.3)$ \\
\hline Testis & 60 & $0(0.0)$ & $60(100.0)$ & $44(73.3)$ & $16(26.7)$ & $0(0.0)$ & $0(0.0)$ & $0(0.0)$ \\
\hline Penis and other male genkal organs & 10 & $0(0.0)$ & $10(100.0)$ & $0(0.0)$ & $4(40.0)$ & $3(30.0)$ & $1(10)$ & $2(20.0)$ \\
\hline Blasder & 126 & $19(15.1)$ & $107(84.9)$ & $1(0.8)$ & $8(6.3)$ & $50(39.7)$ & $62(49.2)$ & $5(4.0)$ \\
\hline Kidney & 106 & $29(27.4)$ & $77(72.6)$ & $2(1.9)$ & $13(12.3)$ & $49(46.2)$ & $38(35.8)$ & $4(3.8)$ \\
\hline Other and ill-defined stes & 4 & $1(25.0)$ & $3(75.0)$ & $0(0.0)$ & $0(0.0)$ & $2(50.0)$ & $2(50.0)$ & $0(0.0)$ \\
\hline Other and unspecified sites & 161 & $120(74.5)$ & $41(25.5)$ & $45(28.0)$ & $62(38.5)$ & $36(22.4)$ & $18(11.2)$ & $0(0.0)$ \\
\hline Eyo & 1 & $0(0.0)$ & $1(100.0)$ & $1(100.0)$ & $0(0.0)$ & $0(0.0)$ & $0(0.0)$ & $0(0.0)$ \\
\hline Brain & 10 & $6(60.0)$ & $4(40.0)$ & $5(50.0)$ & $3(30.0)$ & $1(10.0)$ & $1(10.0)$ & $0(0.0)$ \\
\hline Thyroid gland & 145 & $110(75.9)$ & $35(24.1)$ & $38(26.2)$ & $59(40.7)$ & $33(22.8)$ & $15(10.3)$ & $0(0.0)$ \\
\hline Oner endocrine glands and related structure & 5 & $4(80.0)$ & $1(20.0)$ & $1(20.0)$ & $0(0.0)$ & $2(40.0)$ & $2(40.0)$ & $0(0.0)$ \\
\hline Lymphatic and haematopoietic tissue & 352 & $186(52.8)$ & $166(47.2)$ & $65(18.5)$ & $78(22.2)$ & $107(30.4)$ & $92(26.1)$ & $10(2.8)$ \\
\hline Hodgkin Iymphoma & 74 & $39(52.7)$ & $35(47.3)$ & $42(56.8)$ & $19(25.7)$ & $11(14.9)$ & $2(2.7)$ & $0(0.0)$ \\
\hline Non-Hodgkin lymphoma & 225 & $126(56.0)$ & $99(44.0)$ & $21(9.3)$ & $52(23.1)$ & $72(32.0)$ & $72(32.0)$ & $8(3.6)$ \\
\hline Mutiple myeloma & 35 & $13(37.1)$ & $22(62.9)$ & $0(0)$ & $2(5.7)$ & $17(48.6)$ & $14(40)$ & $2(5.7)$ \\
\hline Leukaemia & 16 & $8(50.0)$ & $8(50.0)$ & $2(12.5)$ & $3(18.8)$ & $7(43.8)$ & $4(25.0)$ & $0(0.0)$ \\
\hline Other and unspecsifed & 2 & $0(0.0)$ & $2(100.0)$ & $0(0.0)$ & $2(100.0)$ & $0(0.0)$ & $0(0.0)$ & $0(0.0)$ \\
\hline Non-skin melanoma & 10 & $8(80.0)$ & $2(20.0)$ & $1(10.0)$ & $1(10.0)$ & $2(20.0)$ & $5(50.0)$ & $1(10.0)$ \\
\hline $\begin{array}{l}\text { Malignant neoplasm without specification of } \\
\text { she }\end{array}$ & 71 & $32(45.1)$ & $39(54.9)$ & $4(5.6)$ & $12(16.9)$ & $38(53.5)$ & $17(23.9)$ & $0(0.0)$ \\
\hline Total & 9578 & $6983(72.9)$ & $2591(27.1)$ & $601(6.3)$ & $2583(27.0)$ & $3756(39.2)$ & $2391(25.0)$ & $243(2.5)$ \\
\hline
\end{tabular}




\section{Acknowledgements}

The authors would like to thank the people who actively contributed to the development of the IEO TR project: Giuseppe Della Porta, Andrea Chiesa, Larissa Dongo, Daniele Dozzo,
Leonardo la Pietra, Gordon McVie, Giulia Peruzzotti, Silvio Pozzi and Nicola Spada. They would also like to thank the following physicians for the clinical counselling: Roberto Biffi, Chiara Casadio, Tommaso De Pas, Roberto Gasparri, Enrica Grosso, Fedro Peccatori, Giuseppe Renne, Bernardo Rocco, Mario Sideri and Giulio Tosti. 


\section{References}

1. Young JL The Hospital-based Tumour Registry Cancer Registration: Principles and Methods (IARC Scientific Publications no 95) ed OM Jensen, DM Parkin, R MacLennan, CS Muir and RG Skeet (Lyon: IARC) pp 177184

2. Cote RA and Robboy S (1980) Progress in medical information management. Systematized nomenclature of medicine (SNOMED) JAMA 243 756-62 PMID 6986000 doi: 10.1001/jama.243.8.756
3. World Health Organization (2007) WHO International Classification of Diseases, 10th revision (Geneva: WHO) http://www.who.int/classifications/apps/icd/icd10online/ (accessed 23 June 2009)

4. Sobin LH and Wittekind C (2002) TNM Classification of Malignant Tumours New York: Wiley-Liss)

5. Botteri E, Bagnardi V, Rotmensz N, Gentilini O, Disalvatore D, Bazolli B, Luini A and Veronesi U (2010) Analysis of local and regional recurrences in breast cancer after conservative surgery Ann Oncol $21 \quad 723-8$ PMID 19833817 doi:10.1093/annonc/mdp386 


\section{Appendices}

\section{Appendix A: Characteristics broken down by tumour}

type: breast cancer (women only)

Table A1: Characteristics at diagnosis

\begin{tabular}{|c|c|c|}
\hline Classification & No. & $\%$ \\
\hline \multicolumn{3}{|l|}{ Age } \\
\hline$<35$ years & 232 & 4.7 \\
\hline $35-49$ years & 1764 & 35.7 \\
\hline $50-64$ years & 1992 & 40.3 \\
\hline $65+$ years & 954 & 19.3 \\
\hline \multicolumn{3}{|l|}{ Histology } \\
\hline Ductal carcinoma & 3773 & 76.3 \\
\hline Lobular carcinoma & 465 & 9.4 \\
\hline Ductal and lobular carcinoma & 179 & 3.6 \\
\hline Cribriform carcinoma & 119 & 2.4 \\
\hline Carcinoma, NOS & 120 & 2.4 \\
\hline Mucinous adenocarcinoma & 88 & 1.8 \\
\hline Tubular adenocarcinoma & 59 & 1.2 \\
\hline Papilllary carcinoma & 28 & 0.6 \\
\hline Phyllodes tumour & 24 & 0.5 \\
\hline Apocrine adenocarcinoma & 16 & 0.3 \\
\hline Medullary carcinoma & 7 & 0.2 \\
\hline Other & 64 & 1.3 \\
\hline \multicolumn{3}{|l|}{$\mathbf{p} T^{\underline{b}}$} \\
\hline РTO ${ }^{c}$ & 14 & 0.3 \\
\hline pTis $^{\mathrm{C}}$ & 18 & 0.4 \\
\hline pT1 & 2903 & 60.6 \\
\hline pT2 & 1411 & 29.5 \\
\hline pT3 & 202 & 4.2 \\
\hline pT4 & 61 & 1.3 \\
\hline pTX & 180 & 3.8 \\
\hline \multicolumn{3}{|l|}{$\mathrm{pN}^{\underline{\underline{b}}}$} \\
\hline pNO & 2367 & 48.5 \\
\hline $\mathrm{pN} 1$ & 1373 & 28.1 \\
\hline pN2 & 485 & 9.9 \\
\hline pN3 & 437 & 8.9 \\
\hline $\mathrm{pNX}$ & 223 & 4.6 \\
\hline \multicolumn{3}{|l|}{$M$} \\
\hline MO/MX & 4745 & 96.0 \\
\hline M1 & 197 & 4.0 \\
\hline
\end{tabular}




\begin{tabular}{|c|c|c|c|c|}
\hline \multicolumn{3}{|c|}{ Type of Treatment } & \multirow{2}{*}{$\begin{array}{l}\text { No. } \\
177\end{array}$} & \multirow{2}{*}{$\begin{array}{c}\% \\
36.0\end{array}$} \\
\hline \multirow{8}{*}{ Surgery } & \multirow{4}{*}{ RT } & HT & & \\
\hline & & CT & 496 & 10.0 \\
\hline & & $\mathrm{HT}+\mathrm{CT}$ & 927 & 18.8 \\
\hline & & $\begin{array}{l}\text { No } \\
\text { HT/CT }\end{array}$ & 153 & 3.1 \\
\hline & \multirow{4}{*}{ No RT } & HT & 322 & 6.5 \\
\hline & & CT & 145 & 2.9 \\
\hline & & $\mathrm{HT}+\mathrm{CT}$ & 345 & 7.0 \\
\hline & & $\begin{array}{l}\text { No } \\
\text { HT/CT }\end{array}$ & 126 & 2.5 \\
\hline \multirow{8}{*}{$\begin{array}{l}\text { Neoadjuvant } \\
\text { treatment + }\end{array}$} & \multirow{4}{*}{ RT } & HT & 147 & 3.0 \\
\hline & & CT & 106 & 2.1 \\
\hline & & $\mathrm{HT}+\mathrm{CT}$ & 139 & 2.8 \\
\hline & & $\begin{array}{l}\text { No } \\
\text { HT/CT }\end{array}$ & 40 & 0.8 \\
\hline & \multirow{4}{*}{ No RT } & HT & 56 & 1.1 \\
\hline & & CT & 18 & 0.4 \\
\hline & & $\mathrm{HT}+\mathrm{CT}$ & 39 & 0.8 \\
\hline & & $\begin{array}{l}\text { No } \\
\text { HT/CT }\end{array}$ & 13 & 0.3 \\
\hline \multirow{6}{*}{ No surgery } & \multirow{3}{*}{ RT } & HT & 6 & 0.1 \\
\hline & & CT & 24 & 0.5 \\
\hline & & $\mathrm{HT}+\mathrm{CT}$ & 17 & 0.3 \\
\hline & \multirow{3}{*}{ No RT } & HT & 7 & 0.1 \\
\hline & & CT & 20 & 0.4 \\
\hline & & $\mathrm{HT}+\mathrm{CT}$ & 19 & 0.4 \\
\hline
\end{tabular}


Figure A1: Place of surgery

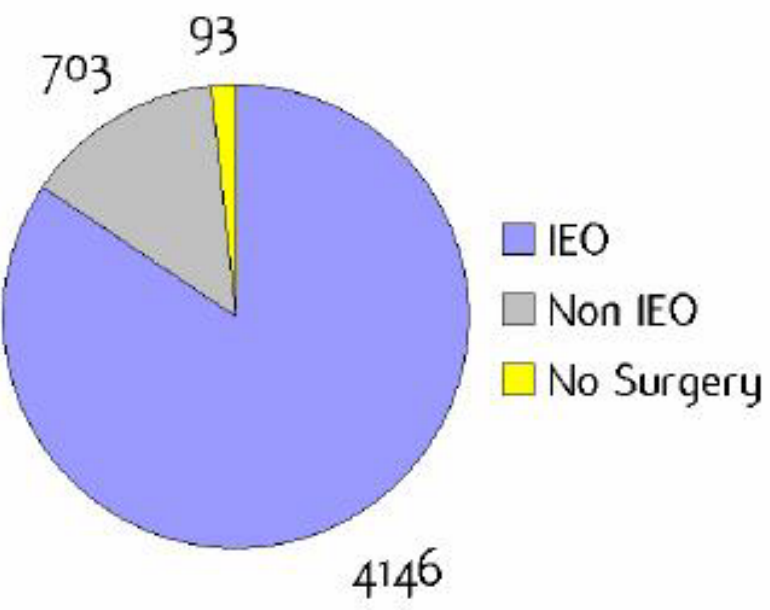

Fig A2: Familiarity for breast and non-breast cancer $\underline{\underline{b}}$

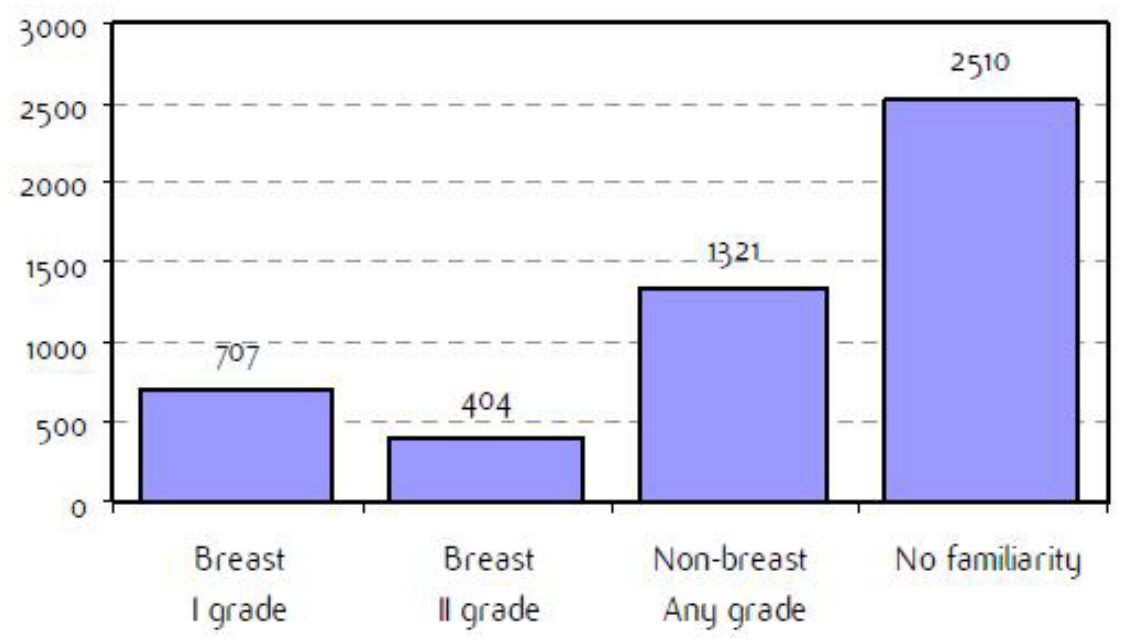

a'Long' record type (at least one treatment in IEO); pathological diagnosis in IEO (at least a slide review in IEO); 'Information is missing for some patients; 'No evidence of primary tumour after neoadjuvant treatment. RT: Radiotherapy; HT: hormonotherapy; CT: chemotherapy and/or monoclonal antibody. NOS: not otherwise specified. 
Appendix B: Characteristics broken down by tumour type: colorectal cancer (468 first invasive primaries treated and diagnosed in IEO ${ }^{a}$ )

Table B1: Characteristics at diagnosis

\begin{tabular}{|c|c|c|}
\hline Classification & No. & $\%$ \\
\hline \multicolumn{3}{|l|}{ Gender } \\
\hline Male & 248 & 53.0 \\
\hline Female & 220 & 47.0 \\
\hline \multicolumn{3}{|l|}{ Age } \\
\hline$<50$ years & 65 & 13.9 \\
\hline $50-59$ years & 117 & 25.0 \\
\hline $60-69$ years & 153 & 32.7 \\
\hline $70-79$ years & 108 & 23.1 \\
\hline $80+$ years & 25 & 5.3 \\
\hline \multicolumn{3}{|l|}{ Histology } \\
\hline Adenocarcinoma & 419 & 89.5 \\
\hline Mucinous adenocarcinoma & 31 & 6.6 \\
\hline Neuroendocrine carcinoma & 5 & 1.1 \\
\hline Carcinoma, NOS & 5 & 1.1 \\
\hline Squamous cell carcinoma & 5 & 1.1 \\
\hline Signet ring cell & & \\
\hline adenocarcinoma & 2 & 0.4 \\
\hline Small cell carcinoma & 1 & 0.2 \\
\hline \multicolumn{3}{|l|}{$\mathrm{pT}^{\underline{\underline{\mathrm{b}}}}$} \\
\hline pT0 & 1 & 0.2 \\
\hline pT1 & 29 & 6.5 \\
\hline pT2 & 94 & 21.2 \\
\hline pT3 & 257 & 58.0 \\
\hline pT4 & 33 & 7.4 \\
\hline pTX & 29 & 6.5 \\
\hline \multicolumn{3}{|l|}{$\mathrm{pN}^{\mathrm{b}}$} \\
\hline pNO & 205 & 44.7 \\
\hline pN1 & 134 & 29.2 \\
\hline pN2 & 79 & 17.2 \\
\hline $\mathrm{pNX}$ & 41 & 8.9 \\
\hline \multicolumn{3}{|l|}{ M } \\
\hline MO/MX & 378 & 80.8 \\
\hline M1 & 90 & 19.2 \\
\hline
\end{tabular}


Table B2: Treatment strategy

\begin{tabular}{|c|c|c|c|c|}
\hline \multicolumn{3}{|c|}{ Type of Treatment } & \multirow{2}{*}{$\begin{array}{c}\text { No. } \\
73\end{array}$} & \multirow{2}{*}{$\begin{array}{c}\% \% \\
15.6\end{array}$} \\
\hline \multirow{6}{*}{ Surgery } & \multirow{2}{*}{ RT } & CT & & \\
\hline & & No CT & 7 & 1.5 \\
\hline & \multirow{4}{*}{ No RT } & HT & 2 & 0.4 \\
\hline & & CT & 195 & 41.7 \\
\hline & & $\mathrm{CT}+\mathrm{HT}$ & 1 & 0.2 \\
\hline & & No CT & 129 & 27.6 \\
\hline \multirow{4}{*}{$\begin{array}{l}\text { Neoadjuvant } \\
\text { treatment + } \\
\text { surgery }\end{array}$} & \multirow[t]{2}{*}{ RT } & CT & 10 & 2.1 \\
\hline & & No CT & 1 & 0.2 \\
\hline & \multirow[t]{2}{*}{ No RT } & CT & 21 & 4.5 \\
\hline & & No CT & 10 & 2.1 \\
\hline \multirow{4}{*}{ No surgery } & \multirow[t]{2}{*}{ RT } & CT & 8 & 1.7 \\
\hline & & No CT & 3 & 0.6 \\
\hline & No & & 8 & \\
\hline & RT & CT & & 1.7 \\
\hline
\end{tabular}

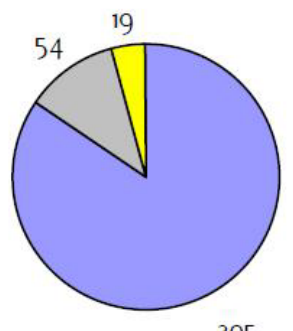

$\square$ IEO

$\square$ Non IEO

$\square$ No Surgery

395

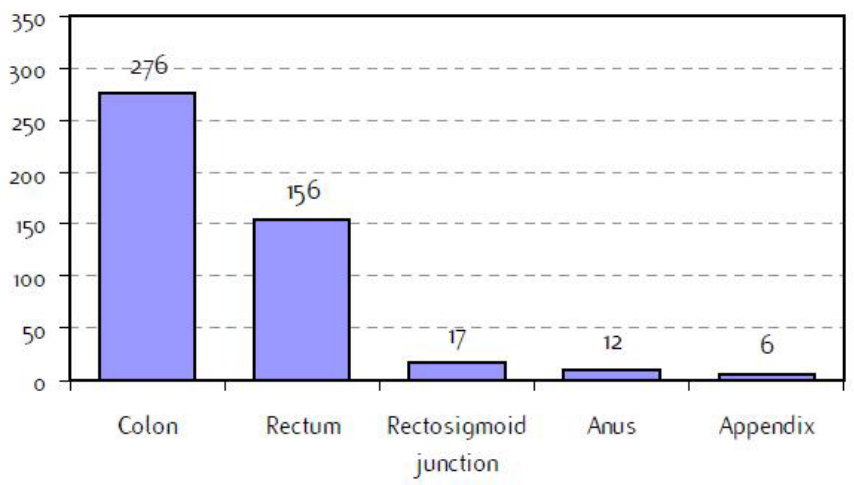

a'Long' record type (at least one treatment in IEO); pathological diagnosis in IEO (at least a slide review in IEO); 'Information is missing for some patients; ${ }^{\circ}$ No evidence of primary tumour after neoadjuvant treatment. RT: radiotherapy; HT: hormonotherapy; CT: chemotherapy and/or monoclonal antibody; NOS: not otherwise specified 
Appendix C: Characteristics broken down by tumour type: lung cancer (627 first invasive primaries treated and diagnosed in IEO')

Table C1: Characteristics at diagnosis

\begin{tabular}{|c|c|c|}
\hline Classification & No. & $\%$ \\
\hline \multicolumn{3}{|l|}{ Gender } \\
\hline Male & 470 & 75.0 \\
\hline Female & 157 & 25.0 \\
\hline \multicolumn{3}{|l|}{ Age } \\
\hline$<35$ years & 13 & 2.1 \\
\hline $35-49$ years & 49 & 7.8 \\
\hline $50-64$ years & 259 & 41.3 \\
\hline $65-79$ years & 293 & 46.7 \\
\hline $80+$ years & 13 & 2.1 \\
\hline \multicolumn{3}{|l|}{ Histology } \\
\hline Adenocarcinoma & 297 & 47.4 \\
\hline $\begin{array}{l}\text { Squamous cell } \\
\text { carcinoma }\end{array}$ & 162 & 25.8 \\
\hline Large cell carcinoma & 89 & 14.2 \\
\hline Small cell carcinoma & 39 & 6.2 \\
\hline Carcinoma, NOS & 40 & 6.4 \\
\hline \multicolumn{3}{|l|}{$\mathrm{pT}^{\underline{\mathrm{b}}}$} \\
\hline pTO & 3 & 0.5 \\
\hline pT1 & 138 & 23.4 \\
\hline pT2 & 231 & 39.1 \\
\hline pT3 & 77 & 13.0 \\
\hline pT4 & 29 & 4.9 \\
\hline pTX & 113 & 19.1 \\
\hline \multicolumn{3}{|l|}{$\mathrm{pN}^{\mathrm{b}}$} \\
\hline pNO & 251 & 50.3 \\
\hline pN1 & 113 & 22.7 \\
\hline $\mathrm{pN} 2$ & 126 & 25.2 \\
\hline pN3 & 9 & 1.8 \\
\hline \multicolumn{3}{|l|}{ M } \\
\hline $\mathrm{MO} / \mathrm{MX}$ & 530 & 84.5 \\
\hline M1 & 97 & 15.5 \\
\hline
\end{tabular}




\section{Table C2: Treatment strategy}

\begin{tabular}{|c|c|c|c|c|}
\hline \multicolumn{3}{|c|}{ Type of Treatment } & \multirow{2}{*}{$\begin{array}{c}\text { No. } \\
1\end{array}$} & \multirow{2}{*}{\begin{tabular}{|c|}
$\%$ \\
0.2 \\
\end{tabular}} \\
\hline \multirow{10}{*}{ Surgery } & \multirow{5}{*}{ RT } & HT & & \\
\hline & & CT & 35 & 5.6 \\
\hline & & $\mathrm{HT}+\mathrm{CT}$ & 3 & 0.5 \\
\hline & & No & & \\
\hline & & HT/CT & 45 & 7.2 \\
\hline & \multirow{5}{*}{ No RT } & HT & 1 & 0.2 \\
\hline & & CT & 25 & 4.0 \\
\hline & & $\mathrm{HT}+\mathrm{CT}$ & 1 & 0.2 \\
\hline & & No & & \\
\hline & & HT/CT & 246 & 39.4 \\
\hline \multirow{4}{*}{$\begin{array}{l}\text { Neoadjuvant } \\
\text { treatment + } \\
\text { Surgery }\end{array}$} & \multirow{2}{*}{ RT } & CT & 20 & 3.2 \\
\hline & & No CT & 40 & 6.4 \\
\hline & \multirow{2}{*}{ No RT } & CT & 8 & 1.3 \\
\hline & & No CT & 79 & 12.6 \\
\hline \multirow[t]{3}{*}{ No surgery } & \multirow{2}{*}{ RT } & CT & 74 & 11.6 \\
\hline & & No CT & 7 & 1.1 \\
\hline & No RT & CT & 42 & 6.7 \\
\hline
\end{tabular}

Figure C1: Place of surgery

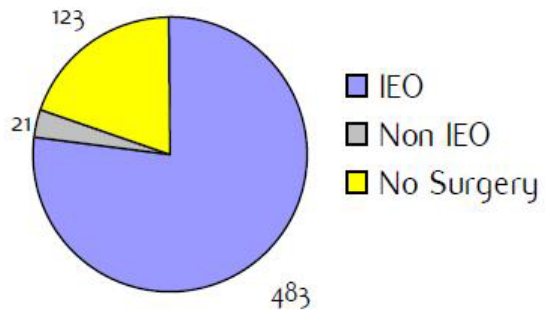

Figure C2: Smoking status at diagnosis ${ }^{\underline{b}}$

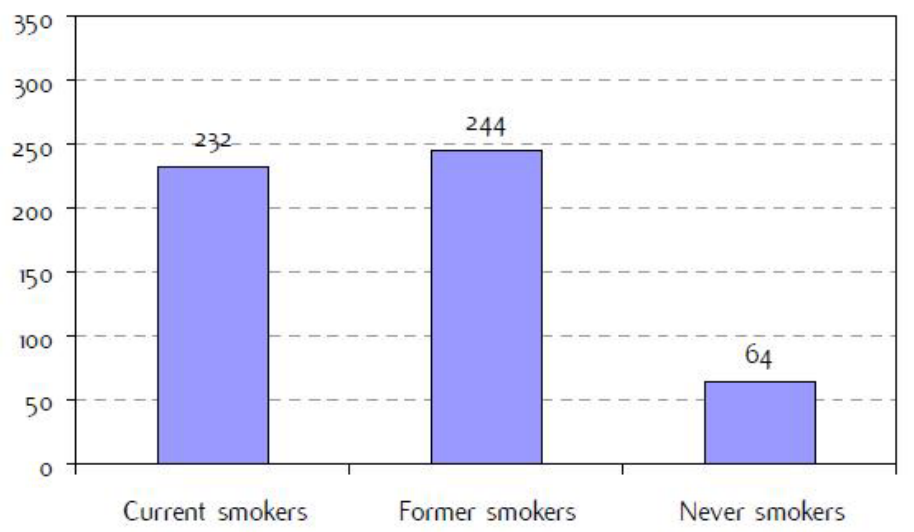

a'Long' record type (at least one treatment in IEO); pathological diagnosis in IEO (at least a slide review in IEO); 'information is missing for some patients; 'No evidence of primary tumour after neoadjuvant treatment. RT: radiotherapy; HT: hormonotherapy; CT: chemotherapy and/or monoclonal antibody. 


\section{Appendix D: Characteristics broken down by tumour} type: non-Hodgkin lymphoma (225 first invasive primaries treated and diagnosed in IEO ${ }^{\mathrm{a}}$ )

Table D1: Characteristics at diagnosis

\begin{tabular}{|c|c|c|}
\hline \multirow{3}{*}{$\begin{array}{l}\text { Classification } \\
\text { Gender } \\
\text { Male }\end{array}$} & \multirow[t]{2}{*}{ No. } & \multirow[t]{2}{*}{$\%$} \\
\hline & & \\
\hline & 99 & 44.0 \\
\hline Female & 126 & 56.0 \\
\hline \multicolumn{3}{|l|}{ Age } \\
\hline$<35$ years & 21 & 9.3 \\
\hline $35-49$ years & 52 & 23.1 \\
\hline $50-64$ years & 72 & 32.0 \\
\hline $65-79$ years & 72 & 32.0 \\
\hline $80+$ years & 8 & 3.6 \\
\hline \multicolumn{3}{|l|}{ Histology ${ }^{2}$} \\
\hline Follicular NHL & 46 & 20.4 \\
\hline Diffuse NHL & 127 & 56.4 \\
\hline Peripheral and cutaneous T-cell L & 11 & 4.9 \\
\hline Other and unspecified types & 41 & 18.2 \\
\hline \multicolumn{3}{|l|}{$\mathbf{p S}^{\varepsilon}$} \\
\hline Stage I & 29 & 15.7 \\
\hline Stage II & 54 & 29.2 \\
\hline Stage III & 19 & 10.3 \\
\hline Stage IV & 83 & 44.8 \\
\hline \multicolumn{3}{|l|}{ Extranodal extension } \\
\hline Present & 118 & 52.4 \\
\hline Absent & 107 & 47.6 \\
\hline \multicolumn{3}{|l|}{ Extranodal site } \\
\hline Stomach & 18 & 15.3 \\
\hline Lip and oral cavity & 11 & 9.3 \\
\hline Skin & 11 & 9.3 \\
\hline Breast & 10 & 8.5 \\
\hline Genitourinary sites & 4 & 3.4 \\
\hline Lung & 4 & 3.4 \\
\hline Major salivary glands & 4 & 3.4 \\
\hline Gynecologic sites & 3 & 2.5 \\
\hline Liver & 3 & 2.5 \\
\hline Soft tissue & 3 & 2.5 \\
\hline Other sites & 11 & 9.3 \\
\hline More sites & 36 & 30.5 \\
\hline
\end{tabular}


Table D2: Treatment strategy

\begin{tabular}{|c|c|c|c|}
\hline \multicolumn{2}{|c|}{ Type of Treatment } & \multirow{3}{*}{$\begin{array}{c}\text { No. } \\
1\end{array}$} & \multirow{3}{*}{$\begin{array}{c}\% \\
0.4\end{array}$} \\
\hline Nodal NHL & & & \\
\hline \multirow{3}{*}{ Surgery } & $\mathrm{RT}+\mathrm{CT}$ & & \\
\hline & Ст & 4 & 1.8 \\
\hline & $\mathrm{RT}+\mathrm{CT}$ & 26 & 11.6 \\
\hline No Surgery & CT & 6 & 33.8 \\
\hline \multicolumn{4}{|c|}{ Extra-nodal NHL } \\
\hline \multirow{4}{*}{ Surgery } & $\mathrm{RT}+\mathrm{CT}$ & 10 & 4.4 \\
\hline & RT & 2 & 0.9 \\
\hline & CT & 11 & 4.9 \\
\hline & No RT/CT & 4 & 1.8 \\
\hline \multirow{4}{*}{ No surgery } & $\mathrm{RT}+\mathrm{CT}$ & 32 & 14.2 \\
\hline & RT & 2 & 0.9 \\
\hline & $\mathrm{RT}+\mathrm{CT}+\mathrm{HT}$ & 1 & 0.4 \\
\hline & Ст & 56 & 24.9 \\
\hline
\end{tabular}

Figure D1: Place of surgery
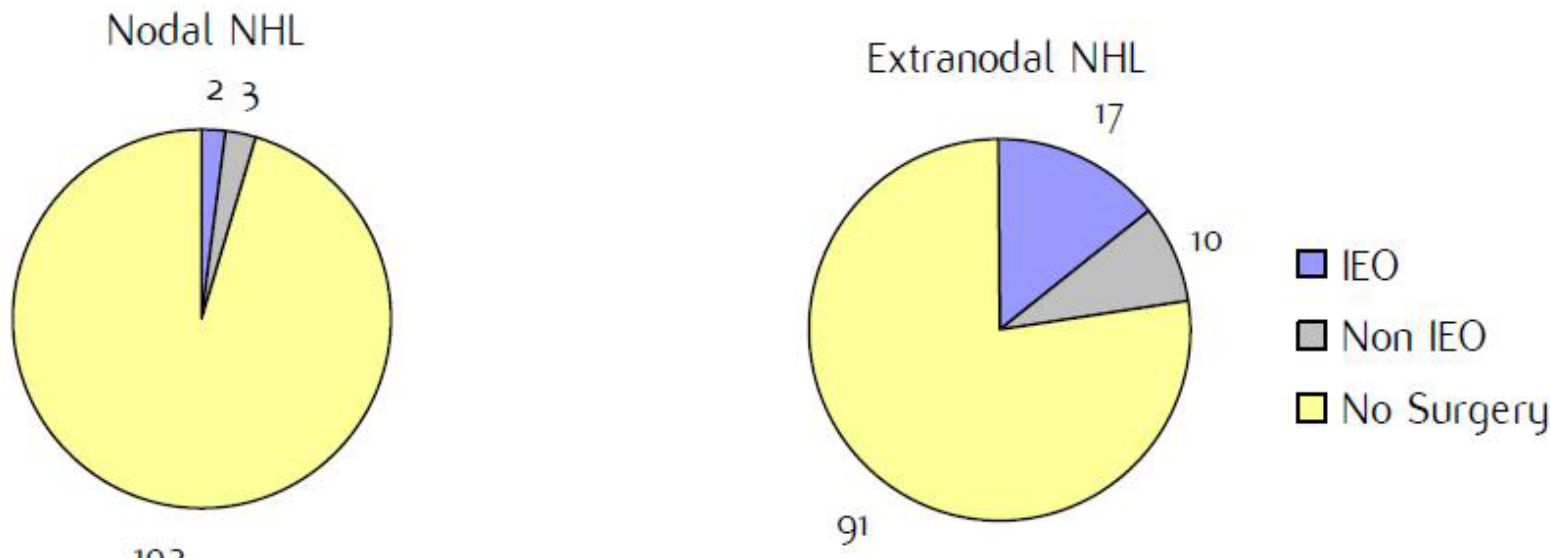

a'Long' record type (at least one treatment in IEO); pathological diagnosis in IEO (at least a slide review in IEO); ${ }^{b}$ WHO classification applied; 'Information is missing for some patients. pS: pathological stage. RT: Radiotherapy; HT: hormonotherapy; CT: chemotherapy and/or monoclonal antibody. NHL: nonHodgkin lymphoma; L: lymphoma. 
Appendix E: Characteristics broken down by tumour type: ovarian cancer (241 first invasive primaries diagnosed and treated in IEO $^{\text {) }}$

Table E1: Characteristics at diagnosis

\begin{tabular}{|c|c|c|}
\hline Classification & No. & $\%$ \\
\hline \multicolumn{3}{|l|}{ Age } \\
\hline$<35$ years & 24 & 10.0 \\
\hline $35-49$ years & 68 & 28.2 \\
\hline $50-64$ years & 108 & 44.8 \\
\hline $65+$ years & 41 & 17.0 \\
\hline \multicolumn{3}{|l|}{ Histology } \\
\hline Serous adenocarcinoma & 98 & 40.7 \\
\hline Endometrioid adenocarcinoma & 37 & 15.4 \\
\hline Borderline malignancy & 34 & 14.1 \\
\hline Adenocarcinoma, NOS & 22 & 9.1 \\
\hline Other type of adenocarcinoma & 15 & 6.2 \\
\hline Clear cell adenocarcinoma & 14 & 5.8 \\
\hline Granulosa cell adenocarcinoma & 7 & 2.9 \\
\hline Mucinous adenocarcinoma & 7 & 2.9 \\
\hline Germ cell tumour & 5 & 2.1 \\
\hline $\begin{array}{l}\text { Transitional cell } \\
\text { adenocarcinoma }\end{array}$ & 2 & 0.8 \\
\hline \multicolumn{3}{|l|}{ FIGO } \\
\hline IA & 35 & 15.6 \\
\hline IB & 5 & 2.2 \\
\hline IC & 26 & 11.6 \\
\hline$\| A$ & 5 & 2.2 \\
\hline IIB & 8 & 3.6 \\
\hline IIC & 9 & 4.0 \\
\hline III & 8 & 3.6 \\
\hline IIIA & 3 & 1.3 \\
\hline IIIB & 6 & 2.7 \\
\hline IIIC & 94 & 41.8 \\
\hline IV & 26 & 11.6 \\
\hline
\end{tabular}


Table E2: Treatment strategy

\begin{tabular}{llcc}
\hline \multicolumn{1}{c}{ Type of Treatment } & No. & $\%$ \\
& HT & 2 & 0.8 \\
& CT & 139 & 57.7 \\
Surgery & CT+HT & 10 & 4.1 \\
& CT+MRT & 4 & 1.7 \\
& No & 54 & 22.4 \\
& HT/CT/MRT & 27 & 11.2 \\
& CT & 2 & 0.8 \\
Neoadjuvant & HT+CT & 1 & 0.4 \\
treatment + & MRT & 1 & 0.4 \\
surgery & No & 1 & 0.4 \\
\hline \multirow{4}{*}{ No surgery } & HT/CT/MRT & CT & \\
\hline
\end{tabular}
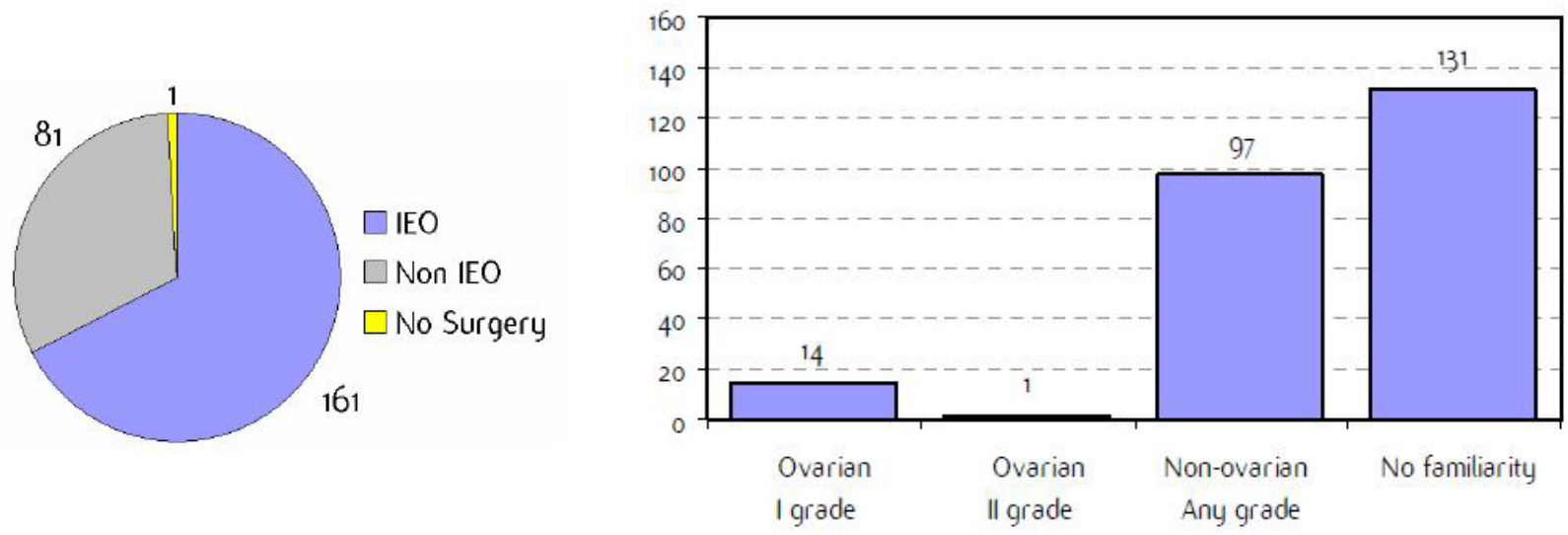

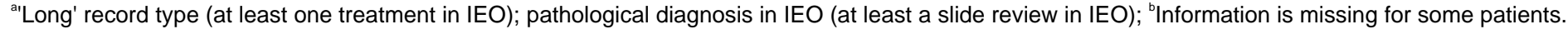
HT: Hormonotherapy; CT: chemotherapy and/or monoclonal antibody; MRT: metabolic radiotherapy; NOS: not otherwise specified. 
Appendix F: Characteristics broken down by tumour type: prostate (376 first invasive primaries treated and diagnosed in IEO $^{\mathrm{a}}$ )

Table F1: Characteristics at diagnosis

\begin{tabular}{|c|c|c|}
\hline Classification & No. & $\%$ \\
\hline \multicolumn{3}{|l|}{ Age } \\
\hline$<65$ years & 173 & 46.0 \\
\hline $65-70$ years & 110 & 29.3 \\
\hline $70-75$ years & 71 & 18.9 \\
\hline $75-80$ years & 17 & 4.5 \\
\hline $80+$ years & 5 & 1.3 \\
\hline \multicolumn{3}{|l|}{ Histology } \\
\hline Acinar adenocarcinoma & 372 & 98.9 \\
\hline Moucinous carcinoma & 2 & 0.5 \\
\hline $\begin{array}{l}\text { Adenosquamous } \\
\text { carcinoma }\end{array}$ & 1 & 0.3 \\
\hline Basaloid carcinoma & 1 & 0.3 \\
\hline \multicolumn{3}{|l|}{$\mathrm{pT}^{\underline{\mathrm{b}}}$} \\
\hline pT2 & 155 & 43.2 \\
\hline pT3 & 112 & 31.2 \\
\hline pT4 & 11 & 3.1 \\
\hline pTx & 81 & 22.6 \\
\hline \multicolumn{3}{|l|}{$\mathrm{pN}^{\mathrm{b}}$} \\
\hline pNO & 154 & 42.5 \\
\hline $\mathrm{pN} 1$ & 14 & 3.9 \\
\hline $\mathrm{pNx}$ & 194 & 53.6 \\
\hline \multicolumn{3}{|l|}{ M } \\
\hline $\mathrm{MO} / \mathrm{Mx}$ & 365 & 97.0 \\
\hline M1 & 11 & 3.0 \\
\hline
\end{tabular}


Table F2: Treatment strategy

\begin{tabular}{|c|c|c|c|c|}
\hline \multicolumn{3}{|c|}{ Type of Treatment } & No. & $\%$ \\
\hline \multirow{8}{*}{ Surgery } & \multirow{4}{*}{ RT } & HT & 37 & 9.8 \\
\hline & & CT & 1 & 0.3 \\
\hline & & $\mathrm{HT}+\mathrm{CT}$ & 4 & 1.1 \\
\hline & & No HT/CT & 48 & 12.8 \\
\hline & \multirow{4}{*}{ No RT } & HT & 31 & 8.2 \\
\hline & & CT & 0 & 0.0 \\
\hline & & $\mathrm{HT}+\mathrm{CT}$ & 2 & 0.5 \\
\hline & & No HT/CT & 174 & 46.3 \\
\hline \multirow{7}{*}{ No surgery } & \multirow{4}{*}{ RT } & HT & 38 & 10.1 \\
\hline & & CT & 0 & 0.0 \\
\hline & & $\mathrm{HT}+\mathrm{CT}$ & 5 & 1.3 \\
\hline & & No HT/CT & 30 & 8.0 \\
\hline & \multirow{3}{*}{ No RT } & HT & 6 & 1.6 \\
\hline & & CT & 0 & 0.0 \\
\hline & & $\mathrm{HT}+\mathrm{CT}$ & 0 & 0.0 \\
\hline
\end{tabular}

Figure F1: Place of surgery

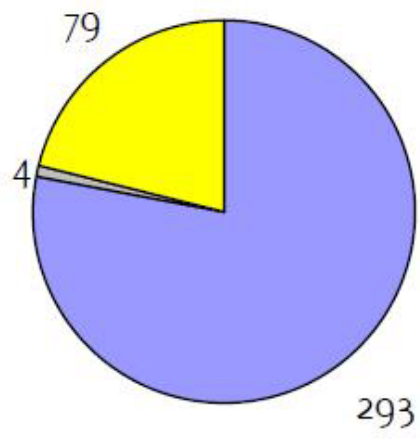

Figure F2: Familiarity for prostatic and non-prostatic cancer

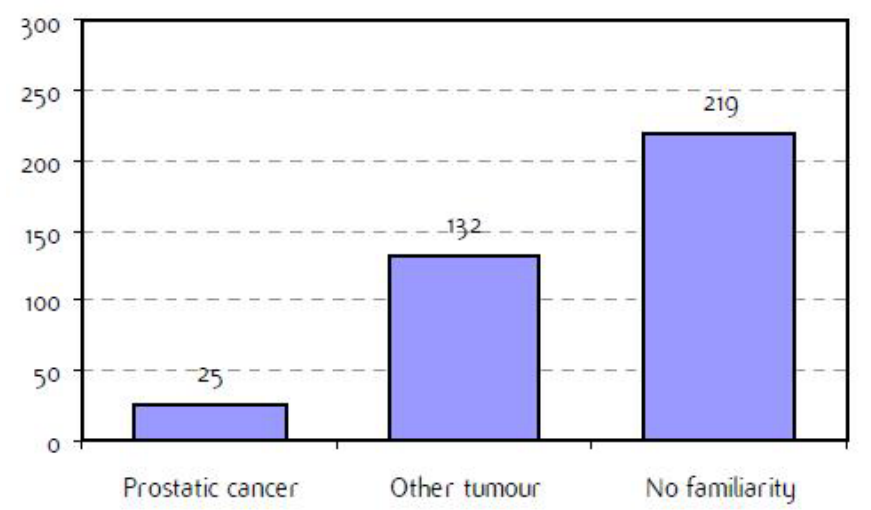

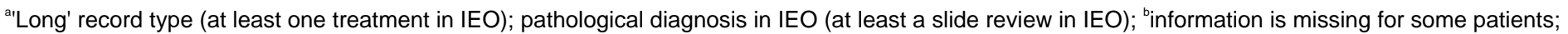
RT: radiotherapy; HT: hormonotherapy; CT: chemotherapy and/or monoclonal antibody. NOS: not otherwise specified. 
Appendix G: Characteristics broken down by tumour type: sarcoma (161 first invasive primaries treated and diagnosed in IEO')

Table G1: Characteristics at diagnosis

\begin{tabular}{|c|c|c|}
\hline Classification & No. & $\%$ \\
\hline \multicolumn{3}{|l|}{ Gender } \\
\hline Male & 76 & 47.2 \\
\hline Female & 85 & 52.8 \\
\hline \multicolumn{3}{|l|}{ Age } \\
\hline$<35$ years & 16 & 9.9 \\
\hline $35-49$ years & 50 & 31.1 \\
\hline $50-64$ years & 42 & 26.1 \\
\hline $65+$ years & 53 & 32.9 \\
\hline \multicolumn{3}{|l|}{ Histology } \\
\hline Sarcoma, NOS & 40 & 24.8 \\
\hline Leiomyosarcoma & 31 & 19.3 \\
\hline Liposarcoma & 25 & 15.5 \\
\hline Fibrosarcoma & 18 & 11.2 \\
\hline Carcinosarcoma & 11 & 6.8 \\
\hline Synovial sarcoma & 8 & 5.0 \\
\hline Angiosarcoma & 6 & 3.7 \\
\hline Epithelioid sarcoma & 5 & 3.1 \\
\hline Kaposi sarcoma & 3 & 1.9 \\
\hline Chondrosarcoma & 2 & 1.2 \\
\hline $\begin{array}{l}\text { Primitive neuroectodermal } \\
\text { tumour }\end{array}$ & 2 & 1.2 \\
\hline Rhabdomyosarcoma & 2 & 1.2 \\
\hline Other & 8 & 5.0 \\
\hline \multicolumn{3}{|l|}{$\begin{array}{l}\text { Specific } \\
\text { site }\end{array}$} \\
\hline Viscerals organs & 44 & 27.3 \\
\hline Trunk & 33 & 20.5 \\
\hline Female genital organs & 24 & 14.9 \\
\hline Lower extremity & 24 & 14.9 \\
\hline Retroperitoneum & 16 & 9.9 \\
\hline Head and neck & 8 & 5 \\
\hline Upper extremity & 8 & 5 \\
\hline Male genital organs & 4 & 2.5 \\
\hline \multicolumn{3}{|l|}{ M } \\
\hline MO/MX & 152 & 94.4 \\
\hline M1 & 9 & 5.6 \\
\hline
\end{tabular}


Table G2: Treatment strategy

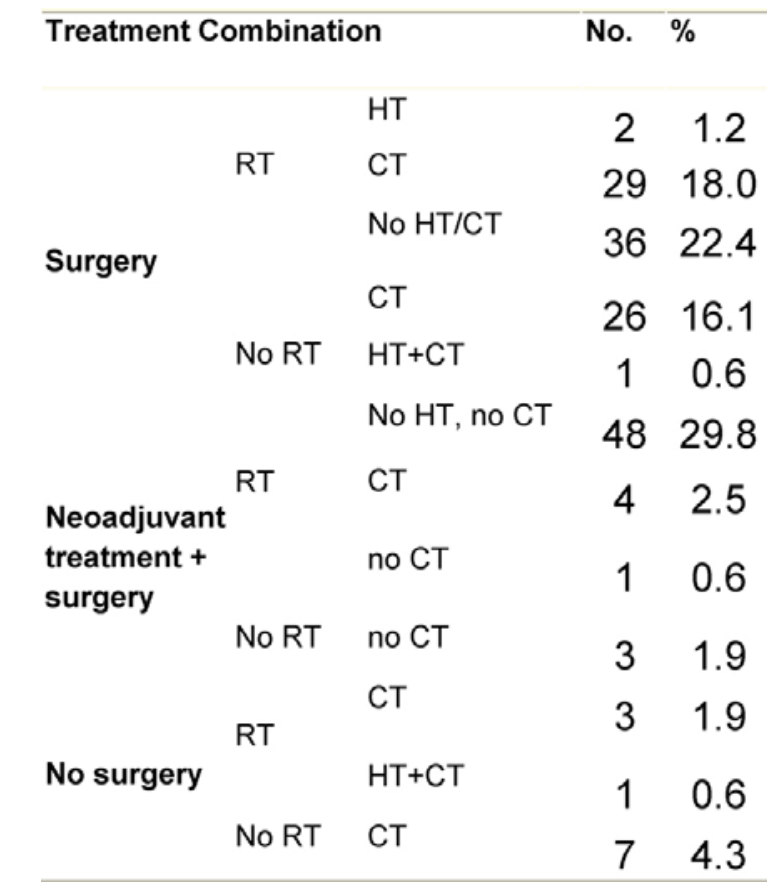

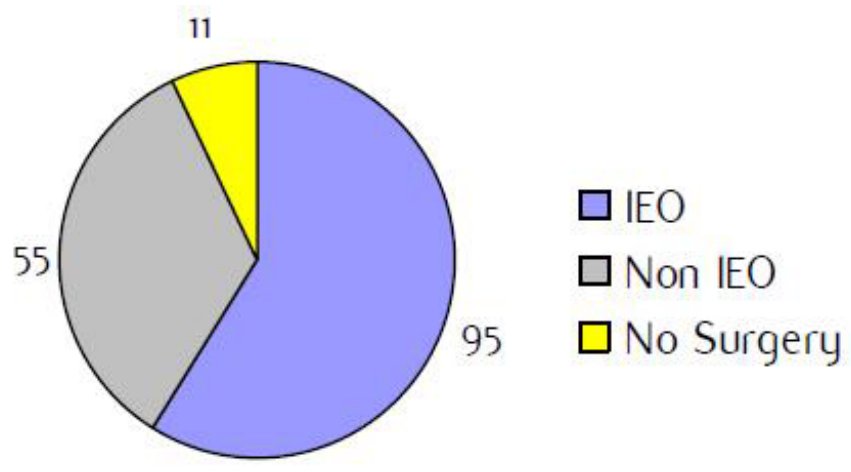

Figure G1: Place of surgery

a'Long' record type (at least one treatment in IEO); pathological diagnosis in IEO (at least a slide review in IEO); 'information is missing for some patients. RT: Radiotherapy; HT: hormonotherapy; CT: chemotherapy and/or monoclonal antibody; NOS: not otherwise specified. 
Appendix H: Characteristics broken down by tumour type: skin melanoma (370 first invasive primaries diagnosed and treated in IEO ${ }^{\mathrm{a}}$ )

Table H1: Characteristics at diagnosis

\begin{tabular}{|c|c|c|}
\hline Classification & No. & $\%$ \\
\hline \multicolumn{3}{|l|}{ Gender } \\
\hline Male & 179 & 48.4 \\
\hline Female & 191 & 51.6 \\
\hline \multicolumn{3}{|l|}{ Age } \\
\hline$<35$ years & 55 & 14.6 \\
\hline $35-49$ years & 127 & 34.3 \\
\hline $50-64$ years & 106 & 28.6 \\
\hline $65+$ years & 82 & 22.2 \\
\hline \multicolumn{3}{|l|}{ Specific site of skin } \\
\hline Skin of head and neck & 26 & 7.0 \\
\hline Skin of upper extremity & 44 & 11.9 \\
\hline Skin of trunk & 158 & 42.7 \\
\hline Skin of lower extremity & 124 & 33.5 \\
\hline Skin, NOS & 18 & 4.9 \\
\hline \multicolumn{3}{|l|}{ Histology } \\
\hline Superficial spreading & 260 & 70.3 \\
\hline Nodular & 56 & 15.1 \\
\hline Malignant, NOS & 36 & 9.7 \\
\hline Acral lentiginous & 9 & 2.4 \\
\hline Lentigo maligna & 8 & 2.2 \\
\hline Desmoplastic & 1 & 0.3 \\
\hline \multicolumn{3}{|l|}{$p T^{\underline{b}}$} \\
\hline pT1 & 68 & 18.7 \\
\hline pT2 & 71 & 19.5 \\
\hline pT3 & 173 & 47.5 \\
\hline pT4 & 33 & 9.1 \\
\hline pTX & 19 & 5.2 \\
\hline \multicolumn{3}{|l|}{$\mathrm{pN}^{\mathrm{b}}$} \\
\hline pNO & 215 & 60.1 \\
\hline $\mathrm{pN} 1$ & 58 & 16.2 \\
\hline pN2 & 5 & 1.4 \\
\hline pN3 & 1 & 0.3 \\
\hline $\mathrm{pNX}$ & 79 & 22.1 \\
\hline \multicolumn{3}{|l|}{$M$} \\
\hline MO/MX & 348 & 94.1 \\
\hline M1 & 22 & 5.9 \\
\hline
\end{tabular}


Table H2: Treatment strategy

\begin{tabular}{|c|c|c|c|c|}
\hline \multicolumn{3}{|c|}{ Type of Treatment } & No. & $\%$ \\
\hline \multirow{2}{*}{\multicolumn{2}{|c|}{ RT }} & $\mathrm{HT}+\mathrm{CT}$ & 6 & 1.6 \\
\hline & & No $\mathrm{HT} / \mathrm{CT}$ & 1 & 0.3 \\
\hline \multirow[t]{5}{*}{ Surgery } & & CT & 14 & 3.8 \\
\hline & No RT & $\mathrm{HT}+\mathrm{CT}$ & 1 & 0.3 \\
\hline & & No HT/CT & 270 & 73.0 \\
\hline & & $\mathrm{CT}$ & 9 & 2.4 \\
\hline & RT & $\mathrm{HT}+\mathrm{CT}$ & 1 & 0.3 \\
\hline \multirow{4}{*}{$\begin{array}{l}\text { Surgery + } \\
\text { immunotherapy }\end{array}$} & & No HT/CT & 3 & 0.8 \\
\hline & & $\mathrm{CT}$ & 15 & 4.1 \\
\hline & No RT & $\mathrm{HT}+\mathrm{CT}$ & 1 & 0.3 \\
\hline & & No HT/CT & 49 & 13.2 \\
\hline
\end{tabular}

Figure H1: Place of surgery

Figure H2: Breslow thickness $\underline{b}$
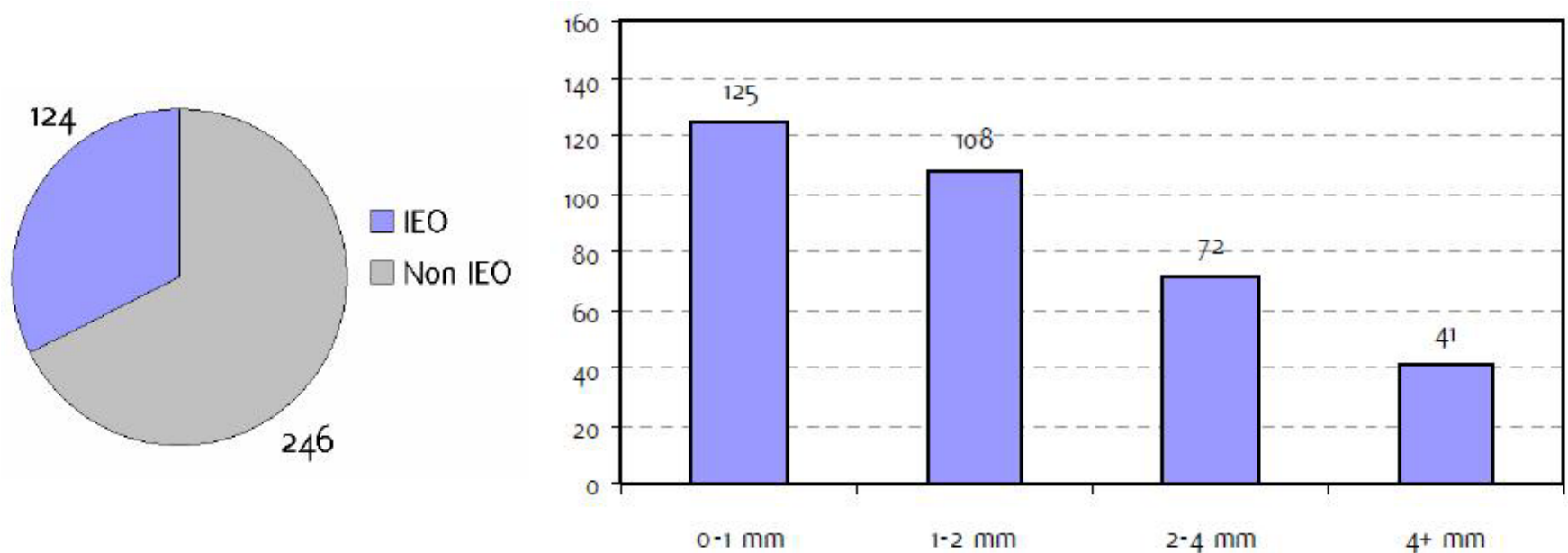

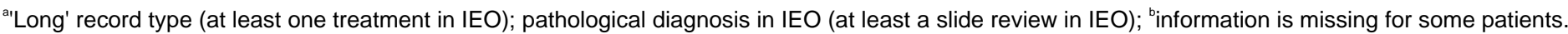
RT: Radiotherapy; HT: hormonotherapy; CT: chemotherapy and/or monoclonal antibody. NOS: not otherwise specified. 
Appendix I: Characteristics broken down by tumour type: tongue (90 first invasive primaries diagnosed and treated in IEO')

Table I1: Characteristics at diagnosis

\begin{tabular}{|c|c|c|}
\hline Classification & No. & $\%$ \\
\hline \multicolumn{3}{|l|}{ Gender } \\
\hline Male & 56 & 62.2 \\
\hline Female & 34 & 37.8 \\
\hline \multicolumn{3}{|l|}{ Age } \\
\hline$<35$ years & 12 & 13.3 \\
\hline $35-49$ years & 14 & 15.6 \\
\hline $50-64$ years & 34 & 37.8 \\
\hline $65+$ years & 30 & 33.3 \\
\hline \multicolumn{3}{|l|}{ Histology } \\
\hline Squamous cell carcinoma & 84 & 93.3 \\
\hline Adenocystic carcinoma & 3 & 3.3 \\
\hline Mucoepidermoid carcinoma & 2 & 2.2 \\
\hline Verrucous carcinoma & 1 & 1.1 \\
\hline \multicolumn{3}{|l|}{$\mathrm{T}^{\underline{\underline{D}}}$} \\
\hline T1 & 26 & 29.9 \\
\hline $\mathrm{T} 2$ & 28 & 32.2 \\
\hline T3 & 8 & 9.2 \\
\hline T4 & 24 & 27.6 \\
\hline TX & 1 & 1.1 \\
\hline \multicolumn{3}{|l|}{$\mathrm{N}^{\underline{2}}$} \\
\hline No & 29 & 32.6 \\
\hline N1 & 18 & 20.2 \\
\hline N2 & 28 & 31.5 \\
\hline N3 & 2 & 2.2 \\
\hline NX & 12 & 13.5 \\
\hline \multicolumn{3}{|l|}{ M } \\
\hline MO/MX & 89 & 98.9 \\
\hline M1 & 1 & 1.1 \\
\hline
\end{tabular}




\begin{tabular}{|l|ll|c|c|}
\hline \multicolumn{2}{|l|}{ Type of Treatment } & & No. & $\%$ \\
\hline \multirow{4}{*}{ Surgery } & RT & CT & 11 & 12.2 \\
\cline { 2 - 5 } & & No CT & 32 & 35.6 \\
\cline { 2 - 5 } & No RT & CT & 0 & 0.0 \\
\cline { 2 - 5 } & & No CT & 37 & 41.1 \\
\hline \multirow{3}{*}{$\begin{array}{l}\text { Neoadjuvant } \\
\text { treatment + }\end{array}$} & RT & CT & 1 & 1.1 \\
\cline { 2 - 5 } surgery & No CT & 1 & 1.1 \\
\cline { 2 - 5 } & No RT & CT & 1 & 1.1 \\
\cline { 2 - 5 } & & No CT & 2 & 2.2 \\
\hline \multirow{4}{*}{ No surgery } & RT & CT & 2 & 2.2 \\
\cline { 2 - 5 } & No CT & 3 & 3.3 \\
\cline { 2 - 5 } & No RT & CT & 0 & 0.0 \\
\cline { 3 - 5 } & & No CT & 0 & 0.0 \\
\hline
\end{tabular}
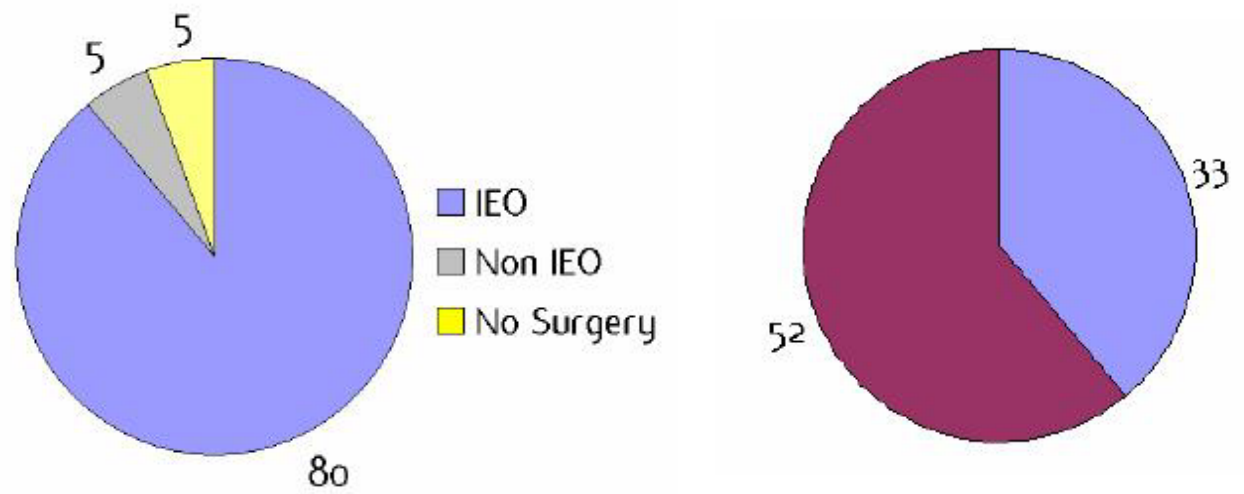

$\square$ Conservative surgery

Demolitive surgery

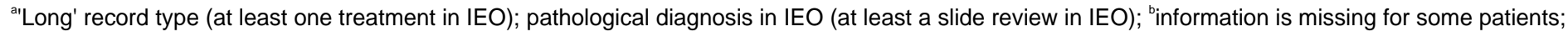
$\mathrm{RT}$ : radiotherapy; HT: hormonotherapy; CT: chemotherapy and/or monoclonal antibody. 